\title{
Upregulation of JMJD2A promotes migration and invasion in bladder cancer through regulation of SLUG
}

\author{
FENGYI WANG, YUANCUI LI, FENGZHI SHAN, QINGLEI ZHANG, \\ LEI WANG, BIN SHENG and GUANGZHOU CHENG \\ Department of Urology Surgery, Tengzhou Central People's Hospital, Tengzhou, \\ Zhaozhuang, Shandong 277500, P.R. China
}

Received November 25, 2018; Accepted March 5, 2019

DOI: $10.3892 /$ or.2019.7246

\begin{abstract}
Jumonji domain-containing protein 2A (JMJD2A) has been identified to promote cell proliferation in bladder cancer; however, it remains undetermined whether JMJD2A regulates cell migration and invasion in bladder cancer. The aim of the present study was to further investigate the roles of JMJD2A in bladder cancer. The expression levels of JMJD2A in bladder cancer tissues and cell lines were established by RT-qPCR assays and western blot analysis. Moreover, by gain- and loss-of-function assays, the effects of JMJD2A on migration and invasion as well as proliferation were investigated in bladder cancer cells. The results revealed that the expression level of JMJD2A was significantly upregulated in bladder cancer tissues and cell lines compared to adjacent non-tumor tissues and a human immortalized bladder urothelial cell line. Kaplan-Meier survival analysis indicated that patients with high JMJD2A expression level had shorter overall survival. Moreover, JMJD2A could promote cell migration and invasion by facilitating epithelial-mesenchymal transition (EMT) in bladder cancer. In addition, it was determined that JMJD2A promoted EMT through regulation of SLUG expression. Collectively, our findings revealed that JMJD2A may act as an oncogene and participate in bladder cancer progression, which provides a promising therapeutic strategy for patients with bladder cancer.
\end{abstract}

\section{Introduction}

Bladder cancer has become the most common tumor of the urinary system globally (1) with almost 350,000-380,000 new cases diagnosed worldwide annually (2). Although a great number of bladder cancer patients have experienced effective treatment including chemotherapy, radiation therapy

Correspondence to: Dr Guangzhou Cheng, Department of Urology Surgery, Tengzhou Central People's Hospital, 181 Xingtan Road, Tengzhou, Zhaozhuang, Shandong 277500, P.R. China

E-mail: cgzhongniu@163.com

Key words: JMJD2A, invasion, EMT, SLUG, bladder cancer and transurethral resection, the prognosis for bladder cancer patients is still unsatisfactory due to advanced stages with distal metastases and frequent recurrences $(2,3)$. Therefore, it is urgent to elucidate the detailed molecular mechanism associated with the tumorigenesis of bladder cancer.

To date, multiple histone methyltransferases and demethylases have been revealed. The JMJD2 family recognizes and demethylates di- and tri-methylated H3K9 and H3K36 (4). JMJD2A is the most studied member of the JMJD2 family, and has been revealed to either activate or suppress gene transcription through its demethylase activity (4-6). Notably, JMJD2A has been revealed to be aberrantly expressed in multiple types of cancer, including breast $(7,8)$, bladder $(9,10)$, lung $(10,11)$ and colon cancer $(12)$. The aim of this study was to explore the function of JMJD2A in human bladder cancer and detail its molecular mechanism.

Previous studies have revealed that epithelial-mesenchymal transition (EMT) is essential for the metastasis of cancers, such as bladder cancer (13). The main characteristic of EMT is cadherin switching and the change of cytoskeleton and cell polarity, which facilitates the motility of cells (14). Moreover, EMT has been demonstrated to be associated with the emergence of chemoresistance in several cancers (14). Additionally, several studies have also revealed that intravesical recurrence is associated with EMT $(15,16)$.

In the present study, our findings revealed that JMJD2A was upregulated in bladder cancer, and high expression of JMJD2A was closely associated with advanced histological grade and poor prognosis of bladder cancer. Moreover, it was revealed that JMJD2A promoted cell migration and invasion through regulation of EMT. Additionally, it was determined that JMJD2A transcriptionally regulated SLUG.

\section{Materials and methods}

Cell culture. Human bladder cancer cell lines (T24 and 5637) and human immortalized bladder urothelial cell line SV-HUC-1 were purchased from the American Type Culture Collection (ATCC; Manassas, VA, USA). T24 and 5637 cells were cultured in McCoy's 5A modified medium (Gibco; Thermo Fisher Scientific, Inc., Waltham, MA, USA) or RPMI-1640 medium (Gibco; Thermo Fisher Scientific, Inc.) with $10 \%$ fetal bovine serum (FBS; Gibco; Thermo Fisher Scientific, Inc). SV-HUC-1 
cells were cultured in F-12K medium (Gibco; Thermo Fisher Scientific, Inc.) supplemented with $10 \%$ FBS. All cells were maintained in a humidified incubator at $37^{\circ} \mathrm{C}$ with $5 \% \mathrm{CO}_{2}$.

Patient tissue specimens. A total of 89 resected specimens from bladder cancer patients were collected for this study. Bladder cancer specimens were compared with paired normal bladder tissue from the same patient. All specimens had been histologically and clinically diagnosed at Tengzhou Central People's Hospital from August 2006 to April 2014, independently, by three experienced pathologists. Prior to surgery, none of the patients had undergone hormone therapy, chemotherapy or radiotherapy. All patients provided written signed informed consent. The study was approved and supervised by the Ethics Committee of Tengzhou Central People's Hospital (Tengzhou, China).

Real-time quantitative polymerase chain reaction (RT-qPCR). Total RNA was extracted from bladder cancer tissues and cell lines using TRIzol reagent (Invitrogen; Thermo Fisher Scientific, Inc.) following the manufacturer's instructions. An RNA sample $(2 \mu \mathrm{g})$ was utilized to synthesize first-strand cDNA using a cDNA Reverse Transcription kit (Takara Bio, Inc., Otsu, Japan). Subsequently, SYBR Green was used to perform a quantitative real-time PCR (qPCR) assay on an Applied Biosystems 7300 Real-Time PCR system (Applied Biosystems; Thermo Fisher Scientific, Inc.). The primer sequences were as follows: JMJD2A forward, 5'-GAA GCCACGAGCATCCTATGA-3' and reverse, 5'-GCGGAA CTCTCGAACAGTCA-3'; E-cadherin forward, 5'-AAACAT CATTGATGCAGACC-3' and reverse, 5'-GATAGATTC TTGGGTTGGGTC-3'; N-cadherin forward, 5'-CAAAGCC TGGAACATATGTG-3' and reverse, 5'-GTTTGAAAGGCC ATATGTGG-3'; SLUG forward, 5'-ACACATACAGTGATT ATTTCCC-3' and reverse, 5'-ACTGTAGTCTTTCCTCTT CAT-3'; SNAIL forward, 5'-TCTAATCCAGAGTTTACCTTC CAG-3' and reverse, 5'-TGAAGTAGAGGAGAAGGACGA-3'; TWIST1 forward, 5'-GTACATCGACTTCCTCTACC-3' and reverse, 5'-GAAACAATGACATCTAGGTCTC-3'; GAPDH forward, 5'-AGAAGGCTGGGGCTCATTTG-3' and reverse, 5'-AGGGGCCATCCACAGTCTTC-3'. GAPDH served as an internal control. The $2^{-\Delta \Delta C q}$ method was utilized to calculate the relative expression of the target gene (17). All experiments were carried out at least three times.

Western blot analysis. Following transfection, cells were collected and lysed in RIPA buffer (Beyotime Institute of Biotechnology, Haimen, China) with protease and phosphatase inhibitors at $4^{\circ} \mathrm{C}$ for $45 \mathrm{~min}$, and the concentration of the samples was quantified using a BCA protein assay kit (Pierce; Thermo Fisher Scientific, Inc.) according to manufacturer's protocol. Western blot analysis was performed according to a previously described procedure (18). A total of $40 \mu \mathrm{g}$ protein was loaded in $10 \%$ SDS-PAGE. Subsequently, proteins were transferred to polyvinylidene difluoride (PVDF) membranes and blocked in 5\% skimmed milk for $1 \mathrm{~h}$ at room temperature. The membranes were incubated with indicated primary antibodies at $4^{\circ} \mathrm{C}$ overnight. After washing with PBST three times, membranes were incubated with HRP conjugated second antibodies at room temperature for $1 \mathrm{~h}$. The following antibodies were used: anti-JMJD2A (dilution 1:1,000; cat. no. ab105953; Abcam, Cambridge, UK); anti-E-cadherin (dilution 1:1,000; cat. no. 14472; Cell Siganling Technology Danvers, MA, USA) and anti-N-cadherin (dilution 1:1,000; cat. no. 13116; Cell Siganling Technology); and anti-SLUG (dilution 1:2,000; cat.no.SAB1305973; Sigma-Aldrich; Merck KGaA,Darmstadt, Germany), anti-TWIST1 (dilution 1:500; cat. no. SAB1409777; Sigma-Aldrich; Merck KGaA), anti-SNAIL (dilution 1:2,000; cat. no. SAB1306281; Sigma-Aldrich; Merck KGaA) and anti- $\beta$-actin (dilution 1:4,000; cat. no. A1978; Sigma-Aldrich; Merck KGaA). The blots were visualized using an enhanced chemiluminescent (ECL) kit (Thermo Fisher Scientific, Inc.). All experiments were carried out at least three times. The densitometry of the blots was quantified by ImageJ (National Institutes of Health, Bethesda, MD, USA).

Wound healing assay. A wound healing assay was carried out to determine the effect of JMJD2A on cell migration ability. Succinctly, a straight wound was scratched using a 20- $\mu 1$ pipette tip when the transfected cells reached $\sim 85-95 \%$ confluence in 12 -well plates. The cells were washed with phosphate-buffered saline (PBS) to remove the detached cells, and the cells were maintained at $37^{\circ} \mathrm{C}$ in a humidified incubator containing $5 \% \mathrm{CO}_{2}$. The images of wound closure were captured at 0 and $48 \mathrm{~h}$ with a digital camera system (Olympus Corp., Tokyo, Japan). All experiments were performed at least three times.

Transwell invasion assay. A Transwell invasion assay was used to examine the effect of JMJD2A on cell invasion ability. In brief, each Transwell chamber (BD Biosciences, Franklin Lakes, NJ, USA) was first coated with $80 \mu$ l Matrigel following the manufacturer's protocol. Approximately $2 \times 10^{5}$ transfected cells were suspended in $500 \mu 1$ serum-free McCoy's 5A modified medium or RPMI-1640 medium and added into the top chamber. Subsequently, $500 \mu 1$ McCoy's 5A modified medium or RPMI-1640 medium containing 10\% FBS was added into the bottom chamber. After incubation for $36 \mathrm{~h}$, the invaded cells were fixed with formaldehyde at room temperature for 10 min and stained with $0.1 \%$ crystal violet at room temperature for $10 \mathrm{~min}$. The non-invaded cells were removed using a cotton swab. The cells were counted in six different fields using a light microscope (Olympus Corp., Tokyo, Japan). All experiments were performed at least three times.

Chromatin immunoprecipitation (ChIP) assay. A chromatin immunoprecipitation (ChIP) assay was performed as previously described (19). Briefly, JMJD2A was overexpressed or knocked down in T24 and 5637 cells, and after transfection for $48 \mathrm{~h}$, the cells were harvested and crosslinked with $1 \%$ formaldehyde for $20 \mathrm{~min}$. Subsequently, the cells were lysed and then sonicated to obtain DNA fragments (200-500 bp in size). Next, the samples were immunoprecipitated with $2 \mu \mathrm{g}$ ChIP-grade antibodies H3K9me2 (cat. no. ab1220; Abcam) overnight at $4^{\circ} \mathrm{C}$, supplemented with protein $\mathrm{G}$ beads. Reversing the cross-links was carried out at $65^{\circ} \mathrm{C}$ overnight. DNA was purified with a Qiagen DNA extraction kit (Qiagen, Inc., Valencia, CA, USA). The immunoprecipitated DNA was purified for RT-qPCR analyses with primers as follows: SLUG forward, 5'-CTGGATTATGCCTCTGTGAT-3' and reverse, 
5'-TGGTATTTATTTGCTGGTAG-3'. All experiments were performed at least three times.

Colony formation assay. Approximately $5 \times 10^{3}$ infected T24 and 5637 cells were plated in 6-well culture plates and cultured with serum-free DMEM. Following culture for 2 weeks at $37^{\circ} \mathrm{C}$ in a $5 \% \mathrm{CO}_{2}$ humidified incubator, the cells were washed with PBS and stained with $0.1 \%$ crystal violet at room temperature for $10 \mathrm{~min}$. Colonies (number of cells $>50$ ) were manually calculated under a light microscope. All experiments were performed at least three times.

Cell counting kit-8 (CCK-8) assay. CCK-8 (Beyotime Institute of Biotechnology) assays were carried out to determine the effect of JMJD2A on cell proliferation. Approximately $4 \times 10^{3}$ transfected cells were suspended and placed in each well of 96-well plates and incubated at $37^{\circ} \mathrm{C}$ in a humidifier incubator containing $5 \% \mathrm{CO}_{2}$. After incubation for $0,24,48$ or $72 \mathrm{~h}$, $20 \mu \mathrm{l}$ of CCK-8 solution was added into the cells of each well and cultured at $37^{\circ} \mathrm{C}$ for $30 \mathrm{~min}$. The optical density (OD) was determined at $450 \mathrm{~nm}$ using an ELISA microplate reader (Bio-Rad Laboratories, Inc., Hercules, CA, USA). All experiments were carried out at least three times.

Statistical analysis. All the data is presented as the mean \pm standard deviation (SD) and analyzed using GraphPad Prism 5.0 (GraphPad Software, La Jolla, CA, USA). Statistical differences among groups were examined using Student's t-test or one-way analysis of variance (ANOVA) followed by Tukey's post hoc test. Kaplan-Meier analysis and log-rank tests were carried out to assess overall survival. $\mathrm{P}<0.05$ was considered to indicate a statistically significant difference.

\section{Results}

JMJD2A is upregulated in bladder cancer tissues. A previous study had indicated that JMJD2A was upregulated in bladder cancer (10). To ascertain whether JMJD2A was upregulated in bladder cancer tissues, RT-qPCR and western blot analysis were conducted in 89 pairs of bladder cancer tissue samples and matched adjacent normal tissue samples. As revealed in Fig. 1A, the expression of JMJD2A was higher in the bladder cancer tissue samples compared with the normal tissue samples (Fig. 1A, P<0.05). The relationship between the expression of JMJD2A and the clinicopathological characteristics of bladder cancer was then assessed. The mean value of JMJD2A mRNA content in tumor cells was used as the standard. Thus, higher values than the standard value were defined as high expression, and lower values than the standard value were defined as low expression. As revealed in Table I, high expression of JMJD2A was positively associated with tumor size and lymphatic invasion as well as high-grade bladder cancer, suggesting that JMJD2A played an essential role in tumor growth and metastasis in bladder cancer. In addition, the survival curve demonstrated that high expression of JMJD2A was associated with shorter overall survival and predicted a poor prognosis (Fig. 1B). Subsequently, the expression of JMJD2A in two bladder cancer cell lines (T24 and 5637) and a human immortalized bladder urothelial cell line (SV-HUC-1), used as a control, was determined. As revealed
Table I. Clinicopathological variables in 89 bladder cancer patients.

\begin{tabular}{|c|c|c|c|c|}
\hline \multirow[b]{2}{*}{ Variables } & \multirow[b]{2}{*}{$\begin{array}{c}\text { No. } \\
(n=89)\end{array}$} & \multicolumn{2}{|c|}{$\begin{array}{l}\text { JMJD2A protein } \\
\text { expression }\end{array}$} & \multirow[b]{2}{*}{ P-value } \\
\hline & & $\begin{array}{c}\text { Low } \\
(n=36)\end{array}$ & $\begin{array}{l}\text { High } \\
(n=53)\end{array}$ & \\
\hline Age (years) & & & & 0.709 \\
\hline$<55$ & 35 & 15 & 20 & \\
\hline$\geq 55$ & 54 & 21 & 33 & \\
\hline Tumor size & & & & 0.019 \\
\hline Small $(\leq 1.5 \mathrm{~cm})$ & 41 & 22 & 19 & \\
\hline Large $(>1.5 \mathrm{~cm})$ & 48 & 14 & 34 & \\
\hline Lymphatic invasion & & & & 0.047 \\
\hline Yes & 41 & 12 & 29 & \\
\hline No & 48 & 24 & 24 & \\
\hline TNM stage & & & & 0.007 \\
\hline I-II & 31 & 24 & 20 & \\
\hline III-IV & 35 & 12 & 33 & \\
\hline
\end{tabular}

JMJD2A expression in bladder cancer tissues from patients was categorized based on tumor stage and analyzed by RT-qPCR.

in Fig. 1C, both the mRNA and protein expression levels of JMJD2A were high in bladder cancer cell lines, T24 and 5637 compared with those in SV-HUC-1 (Fig. 1C, P=0.042). These data indicated that JMJD2A may function as an oncogene in bladder cancer.

JMJD2A promotes bladder cancer cell migration and invasion. To explore the effect of JMJD2A on bladder cancer cells, JMJD2A was first overexpressed or knocked down in T24 and 5637 cells. After confirming the expression of JMJD2A (Fig. 2A), wound healing and Transwell invasion assays were performed to detect whether JMJD2A affected bladder cancer cell migration and invasion. Wound healing assays revealed that the migratory ability of T24 and 5637 cells was significantly enhanced when JMJD2A was overexpressed; whereas the migratory ability of T24 and 5637 cells was decreased when JMJD2A was knocked down (Fig. 2B). Consistent with the wound healing assay result, in Transwell invasion assays, the number of invaded cells in JMJD2A-overexpressed T24 and 5637 was increased; however, the number of invaded cells in JMJD2A-depleted T24 and 5637 was decreased, compared to the control cell group (Fig. 2C). Overall, these results indicated that JMJD2A promoted bladder cancer cell migration and invasion.

Overexpression of JMJD2A facilitates EMT in bladder cancer cells. EMT plays a crucial role in the metastasis of bladder cancer cells (20). To further determine whether JMJD2A promoted bladder cancer cell migration and invasion through regulation of EMT, the expression of EMT-associated genes, including E-cadherin, $\mathrm{N}$-cadherin and vimentin in bladder cancer cells, was detected by RT-qPCR and western blotting. 
A
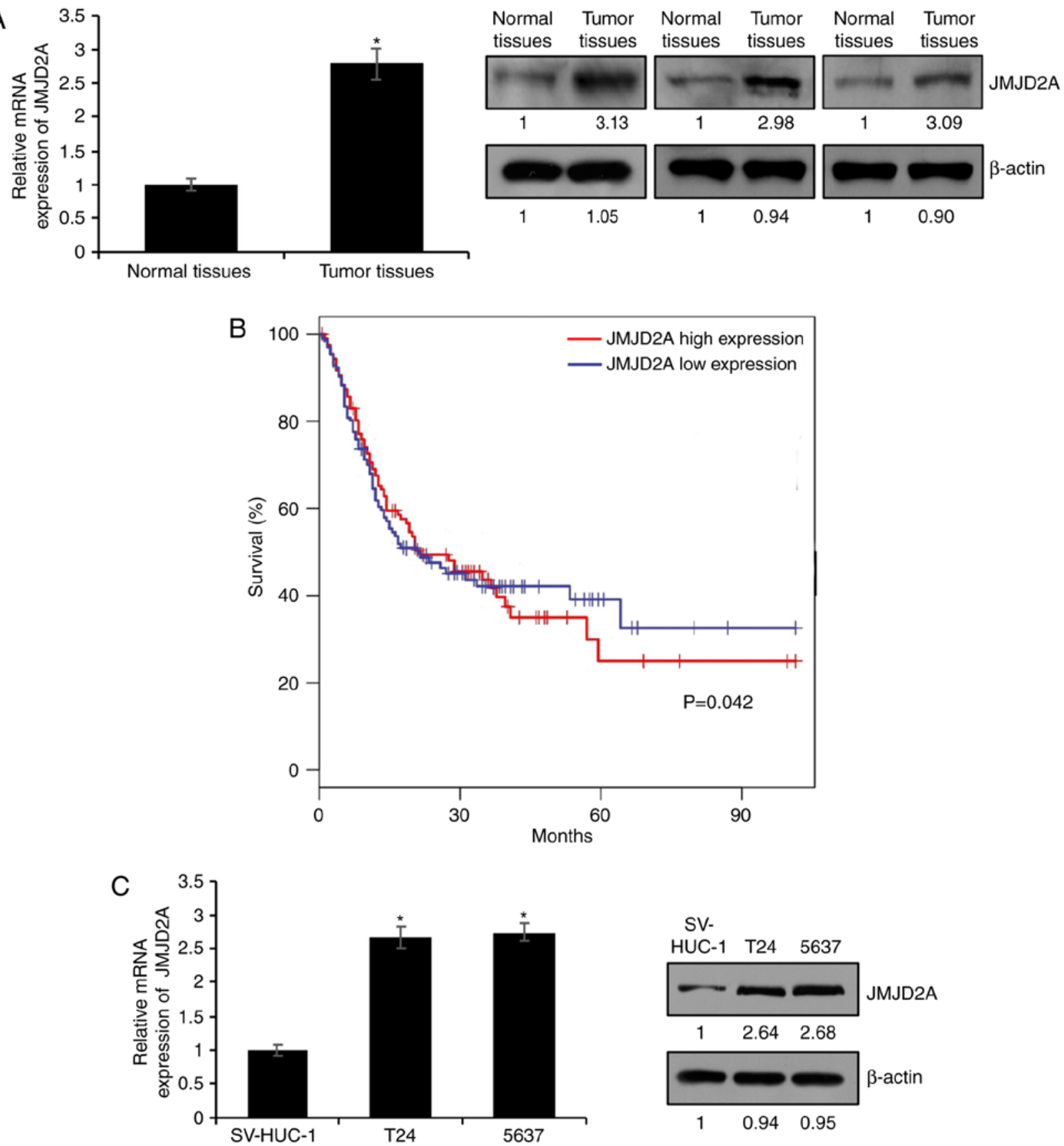

Figure 1. JMJD2A is upregulated in bladder cancer tissues. (A) RT-qPCR and western blotting of JMJD2A were carried out in tumor tissues and adjacent normal tissues. "P<0.05 vs. normal tissues. (B) Kaplan-Meier plots demonstrated that survival was based on the expression of JMJD2A. (C) The expression of JMJD2A in bladder cancer cell lines (T24 and 5637) and a human immortalized bladder urothelial cell line (SV-HUC-1) was observed using RT-qPCR and western blot analyses. "P<0.05 vs. SV-HUC-1.

As revealed in Fig. 3A and B, the expression of N-cadherin and vimentin was upregulated, and in contrast, E-cadherin expression was significantly downregulated upon JMJD2A overexpression in T24 and 5637 cells; however, inhibition of JMJD2A resulted in an increased expression of E-cadherin and a decreased expression of $\mathrm{N}$-cadherin and vimentin (Fig. 3C and D). These findings demonstrated that JMJD2A promoted bladder cancer cell migration and invasion by inactivating the EMT pathway.

$J M J D 2 A$ transcriptionally regulates $S L U G$ in bladder cancer cells. To further decipher the detailed mechanism of JMJD2A on the modulation of EMT, it was next examined whether JMJD2A regulated the expression of EMT-related transcription factors, including SLUG, Twist1, and SNAIL. Notably, it was revealed that the overexpression/knockdown of JMJD2A had little effect on the expression of TWIST1 and SNAIL but affected the expression of SLUG (Fig. 4A and B). These results revealed that JMJD2A regulated EMT possibly through the modulation of SLUG. qChIP analysis was next performed to determine whether JMJD2A regulated this gene by modulating H3K9me2 demethylation at promoter regions. In order to verify this hypothesis, qChIP assay was used with anti-H3K9me2 in JMJD2A overexpression or JMJD2A-depleted T24 or 

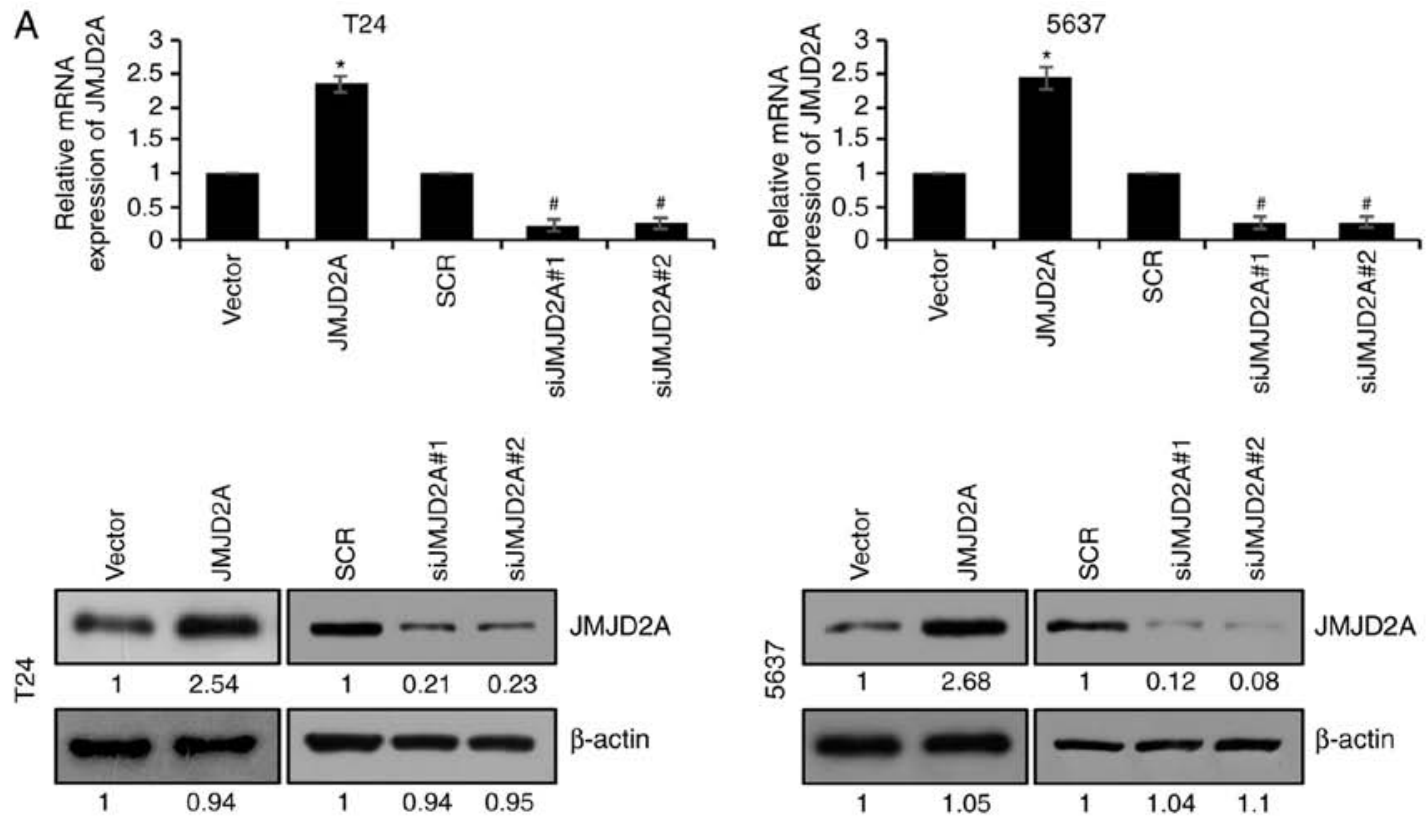

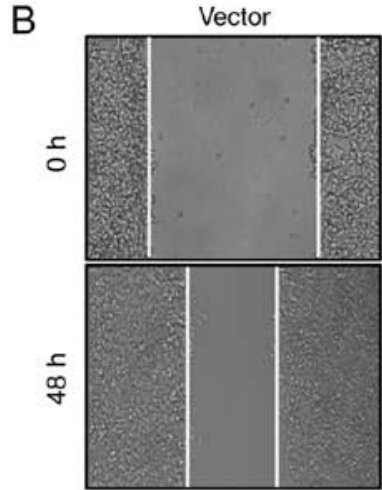

T24

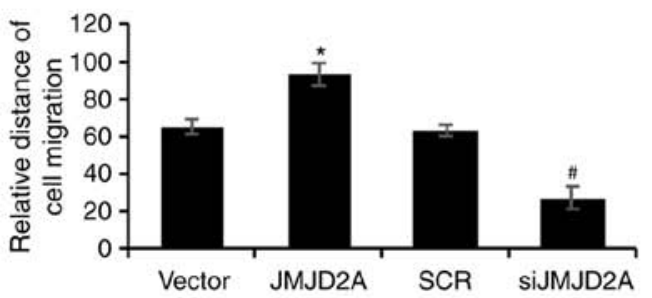

C
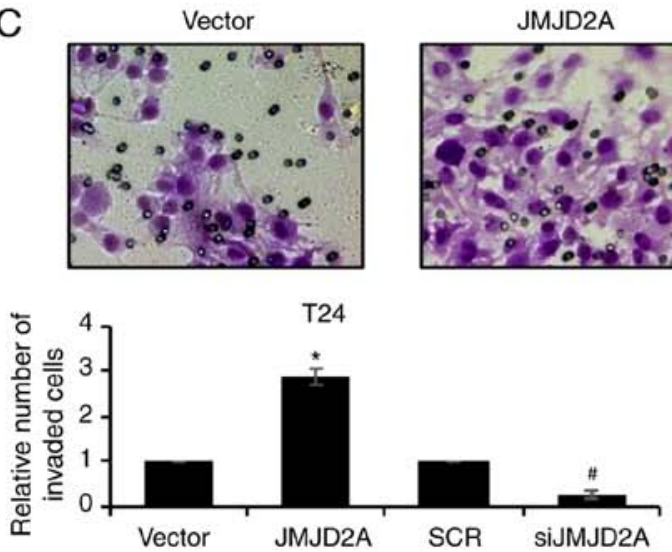
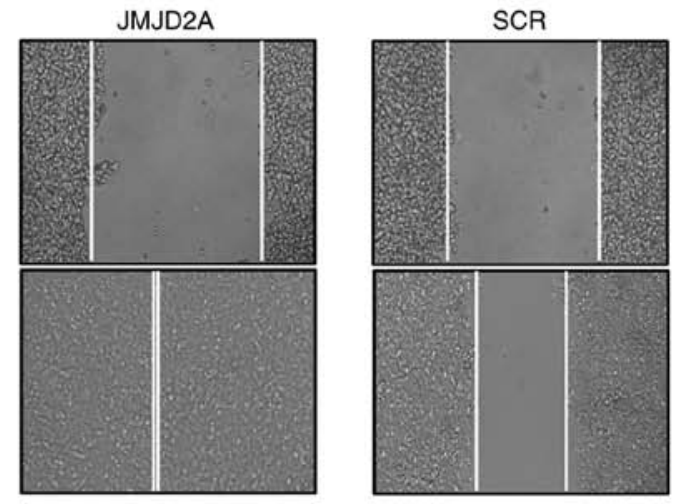

5637
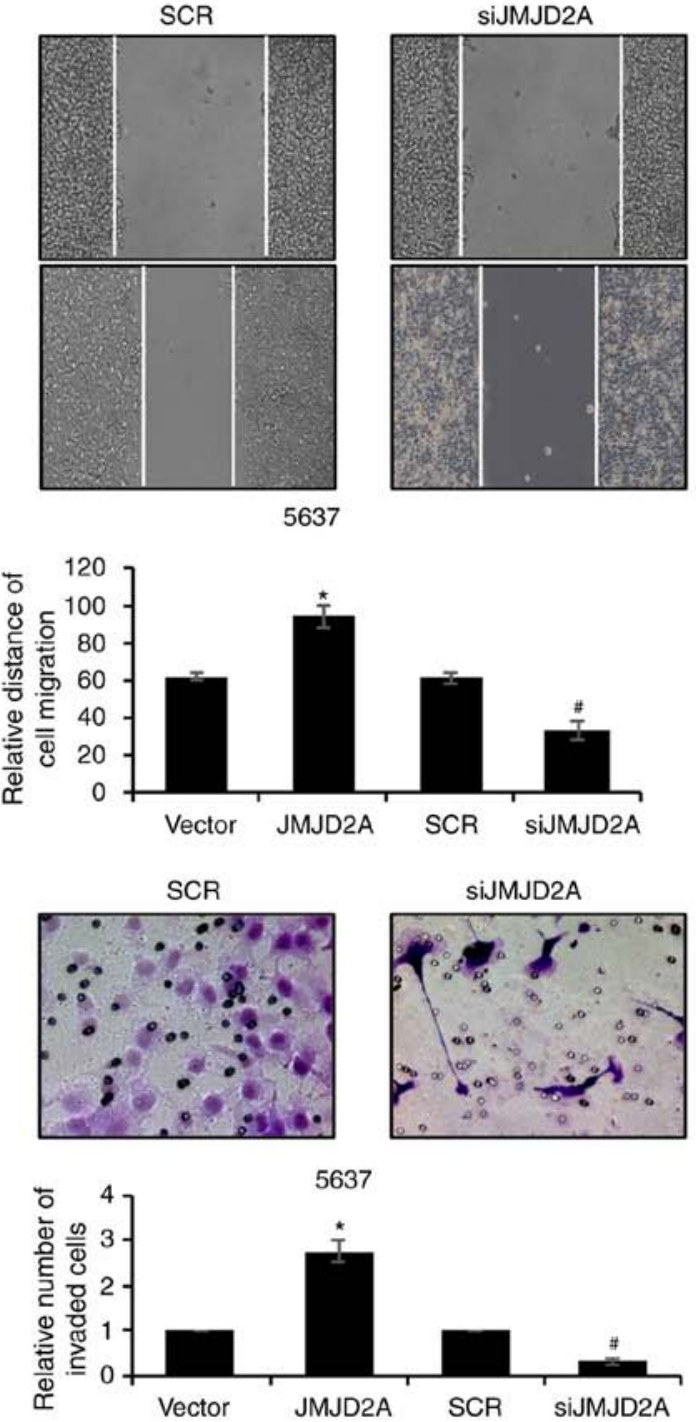

Figure 2. JMJD2A promotes bladder cancer cell migration and invasion. (A) JMJD2A expression levels were determined by RT-qPCR and western blot analyses after treatment of bladder cancer cell lines with either the vector, the FLAG-JMJD2A plasmid or SCR and siJMJD2A. "P<0.05 vs. vector, ${ }^{\text {P }}<0.05$ vs. SCR. (B) Wound healing assays in T24 and 5637 cells expressing either the vector, the FLAG-JMJD2A plasmid or SCR and siJMJD2A. The images represent the experiment in T2 4 cells. ${ }^{*} \mathrm{P}<0.05$ vs. vector, ${ }^{\prime \prime} \mathrm{P}<0.05$ vs. SCR. (C) Transwell invasion assays in T24 and 5637 cells expressing either the vector, the FLAG-JMJD2A plasmid or SCR and siJMJD2A. The images represent the experiment in T24 cells. SCR, scramble siRNA; siJMJD2A, JMJD2A siRNA. ${ }^{*} \mathrm{P}<0.05$ vs. vector, ${ }^{\#} \mathrm{P}<0.05$ vs. SCR. 
A
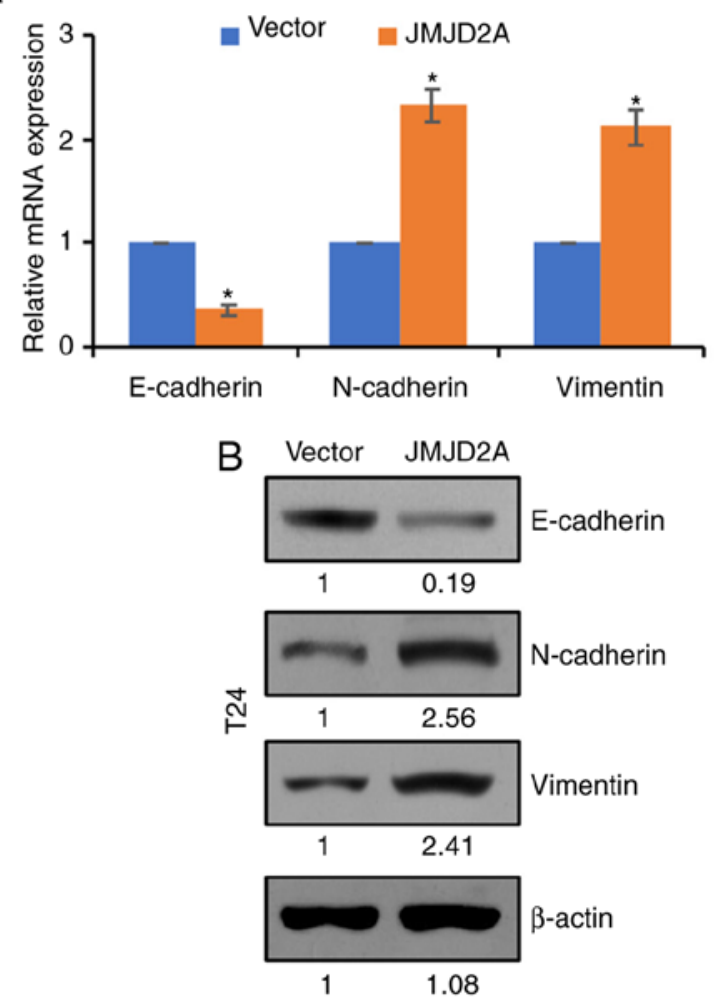

C

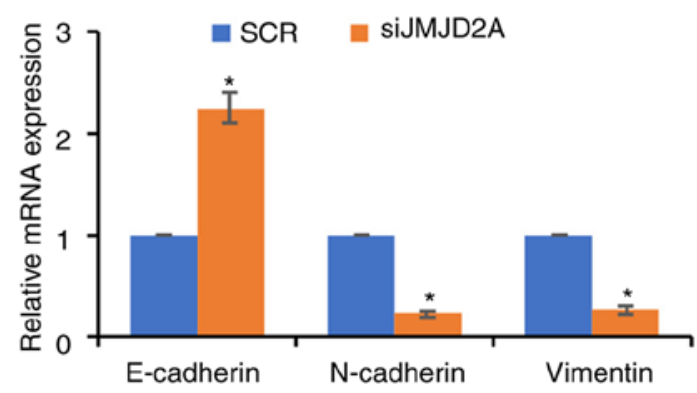

$\mathrm{D}$
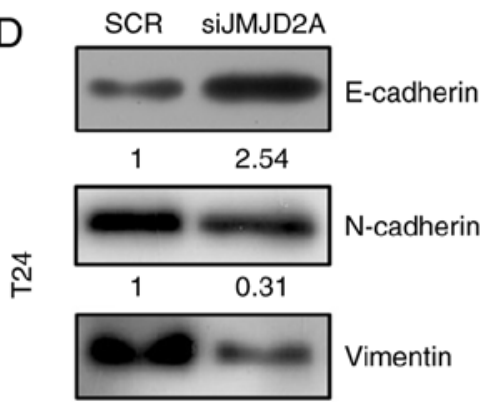

10.15

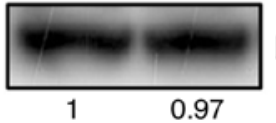

5637
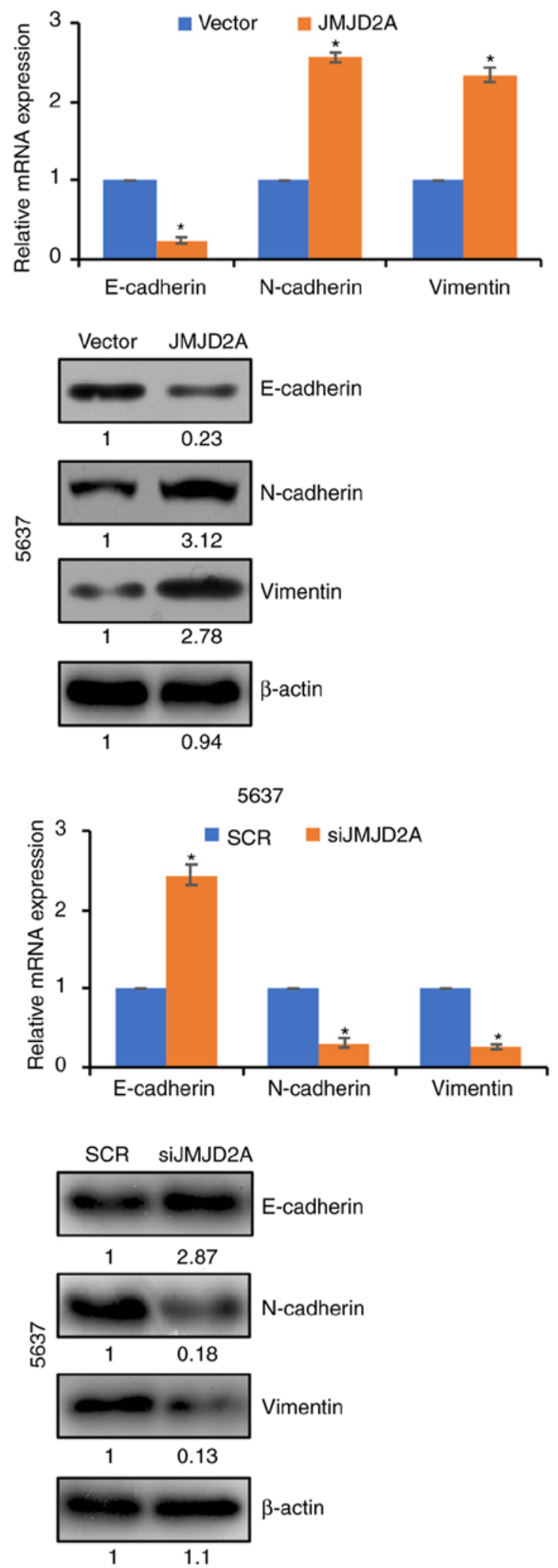

Figure 3. Overexpression of JMJD2A facilitates EMT in bladder cancer cells. (A and B) JMJD2A was overexpressed in T24 and 5637 cells. After transfection for $48 \mathrm{~h}$, RT-qPCR and western blot analyses were performed to assess the effects of JMJD2A on EMT. *P<0.05 vs. vector (C and D) JMJD2A was knocked down in T24 and 5637 cells. After transfection for 48 h, RT-qPCR and western blot analyses were performed to assess the effects of JMJD2A on EMT. EMT, epithelial-mesenchymal transition. ${ }^{*} \mathrm{P}<0.05$ vs. SCR. SCR, scramble siRNA.

5637 cells. The results revealed that $\mathrm{H} 3 \mathrm{~K} 9 \mathrm{me} 2$ at the promoter of $S L U G$ was significantly increased when JMJD2A was knocked down in T24 and 5637 cells; however, H3K9me2 at the promoter of $S L U G$ was significantly decreased when JMJD2A was overexpressed in T24 and 5637 cells (Fig. 4C). These results indicated that $S L U G$ was transcriptionally 
A

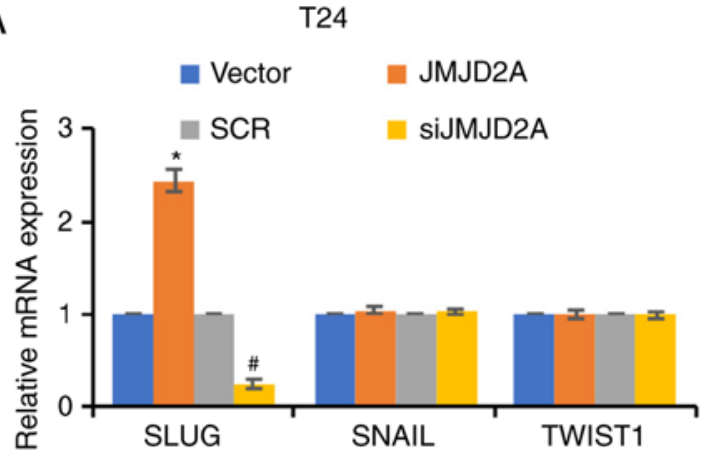

B
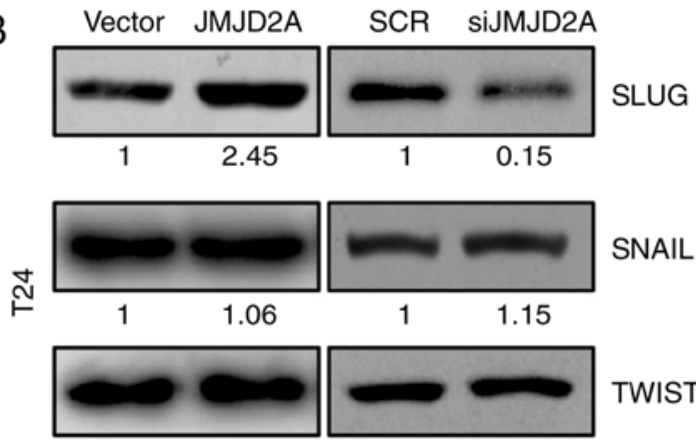

$1 \quad 1.08$

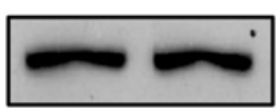

1

C

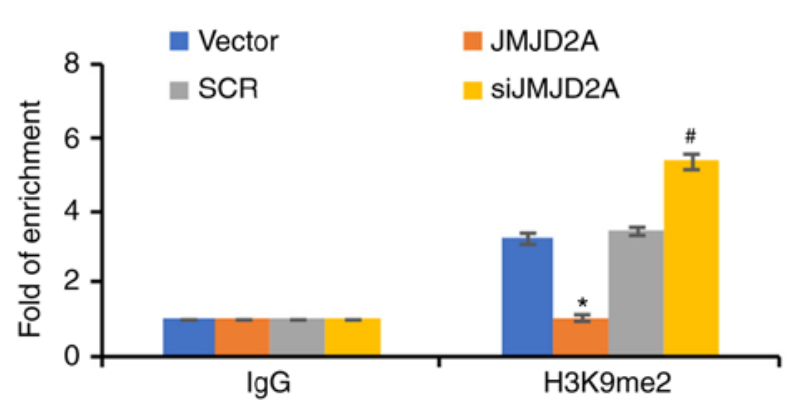

5637
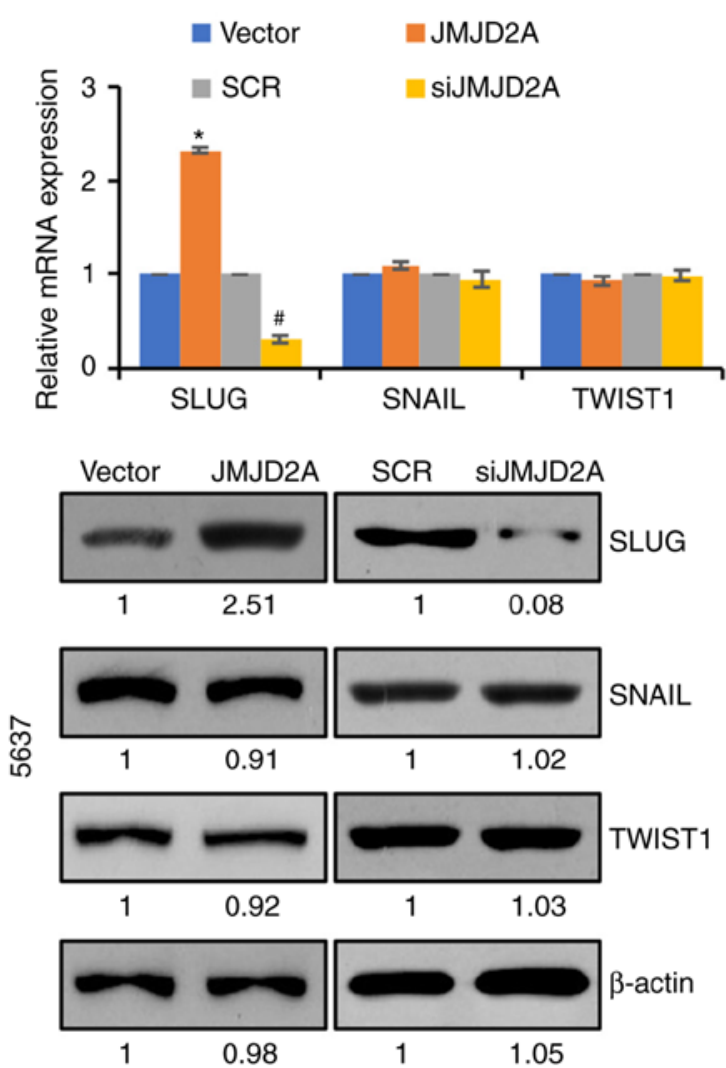

5637

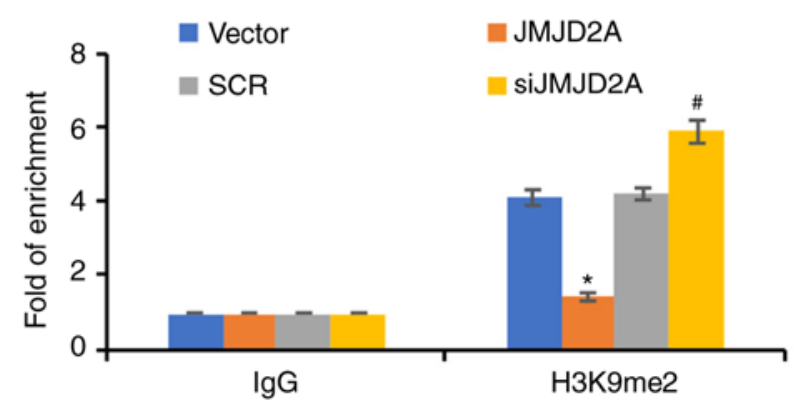

Figure 4. JMJD2A transcriptionally regulates SLUG in bladder cancer cells. (A and B) JMJD2A was overexpressed or knocked down in T24 and 5637 cells. After transfection for $48 \mathrm{~h}$, the effects of JMJD2A on EMT-associated transcription factors, including SNAIL, SLUG and TWIST1 were assessed. *P<0.05 vs. vector, ${ }^{\#} \mathrm{P}<0.05$ vs. SCR. (C) qChIP assay was performed using $\mathrm{H} 3 \mathrm{~K} 9 \mathrm{me} 2$ antibody in JMJD2 overexpressed or depleted cells. ${ }^{*} \mathrm{P}<0.05$ vs. vector, ${ }^{\#} \mathrm{P}<0.05$ vs. SCR. SCR, scramble siRNA.

regulated by JMJD2A through its $\mathrm{H} 3 \mathrm{~K} 9$ demethylase activity. The aforementioned results revealed that JMJD2A transcriptionally activated SLUG expression in bladder cancer.

SLUG overexpression reverses the effects of JMJD2A on migration and invasion in bladder cancer cells. To further investigate whether SLUG was involved in JMJD2A-mediated malignant phenotypes of bladder cancer cells, SLUG was overexpressed in JMJD2A-depleted T24 or 5637 cells. The mRNA and protein levels of SLUG were subsequently established using RT-qPCR and western blot analyses, respectively. As revealed in Fig. 5A and B, co-transfection with JMJD2A siRNA and SLUG plasmids reversed the inhibitory effect on SLUG expression in T24 or 5637 cells compared with transfection with JMJD2A siRNA alone
(Fig. 5A and B). The migration and invasion of these two groups were also compared. As revealed in Fig. 5C and D, overexpression of SLUG reversed the inhibitory effects on migration and invasion of JMJD2A-depleted T24 and 5637 cells (Fig. 5C and D), revealing that SLUG was in fact involved in JMJD2A-mediated malignant phenotypes of bladder cancer cells.

JMJD2A promotes bladder cancer cell proliferation. To further confirm the biological function of JMJD2A, CCK-8 and colony formation assays were performed to identify the roles of JMJD2A on bladder cancer cell proliferation. As revealed ectopic expression of JMJD2A promoted the cell proliferation rate, compared with vector control group; however, inhibition of JMJD2A suppressed the cell proliferation rate compared 
A

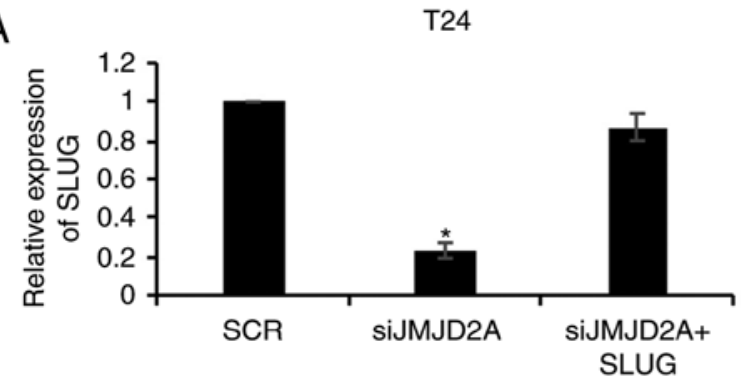

B
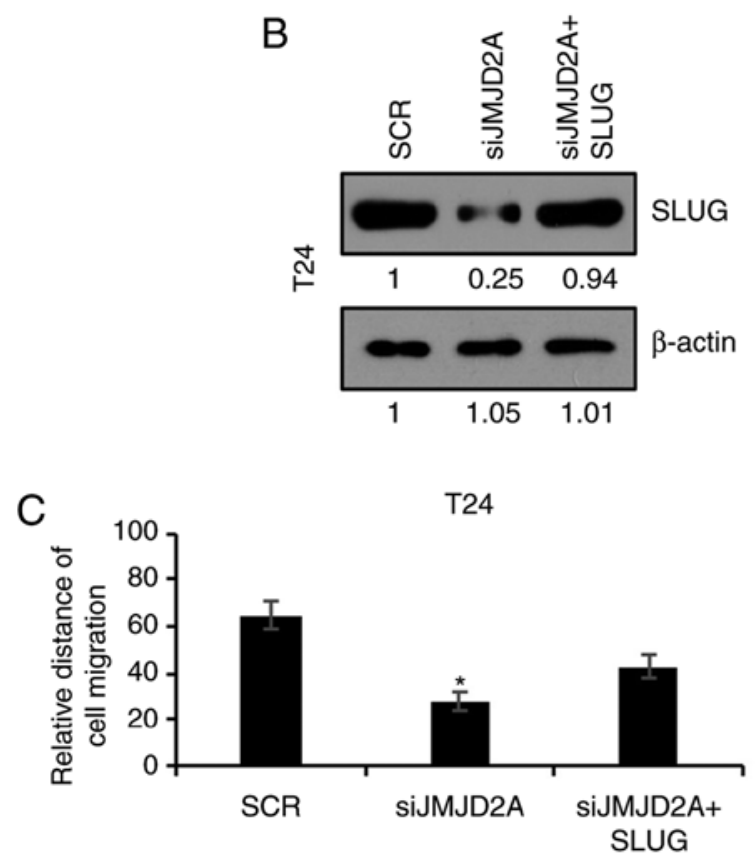

D

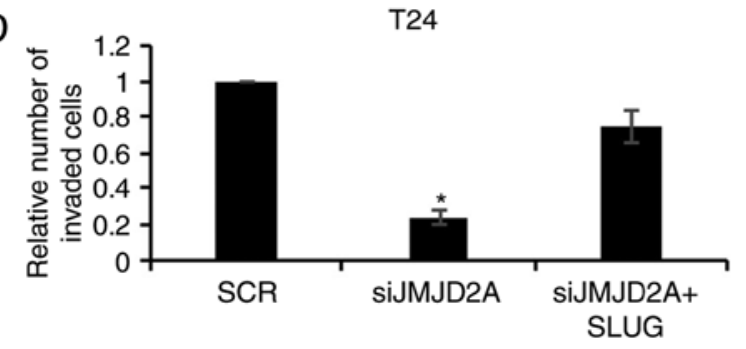

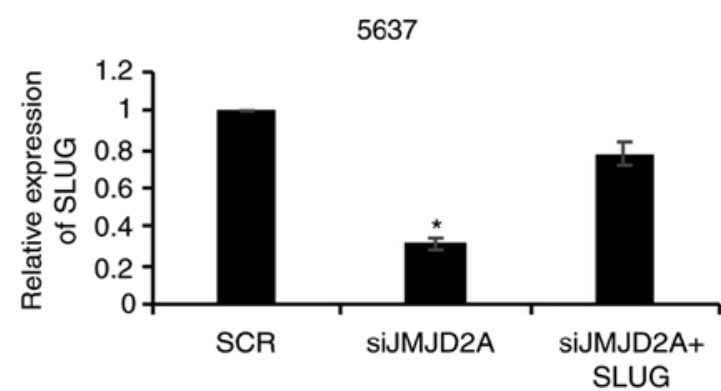
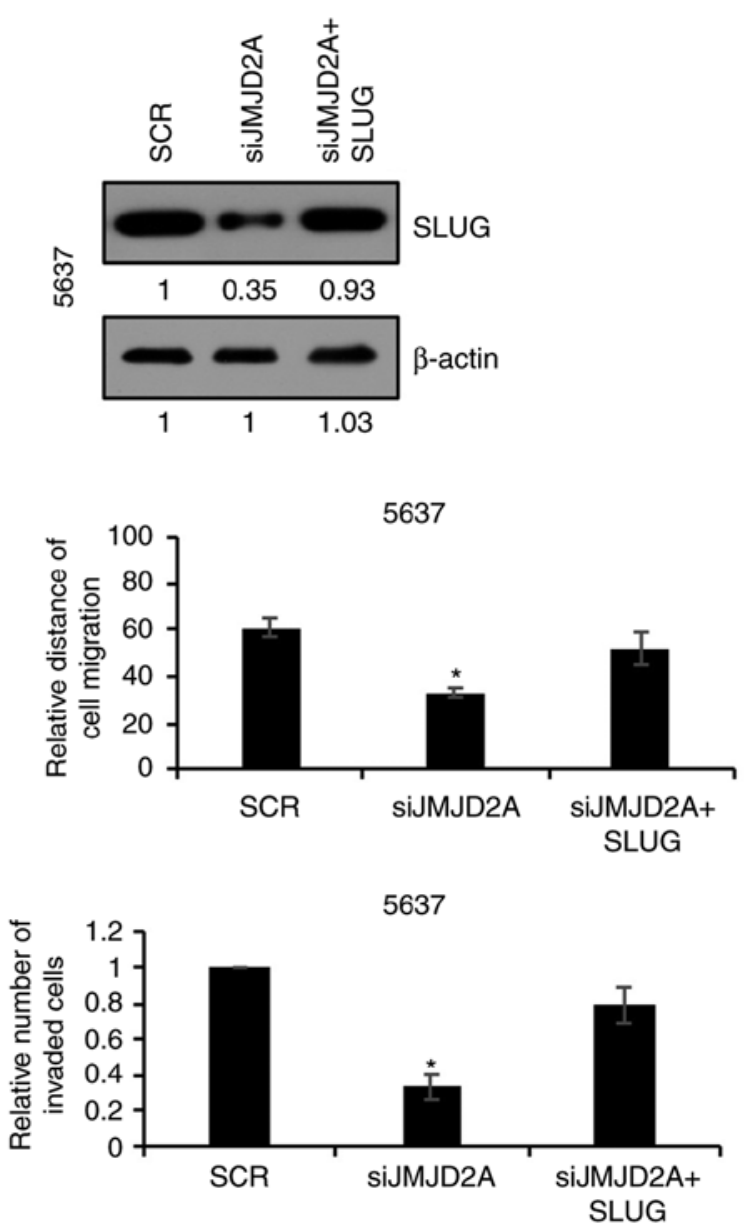

Figure 5. SLUG overexpression reverses the effects of JMJD2A on migration and invasion in bladder cancer cells. (A and B) SLUG was overexpressed in JMJD2A-depleted T24 and 5637 cells, and the expression of SLUG was determined using RT-qPCR and western blot analyses. *P<0.05 vs. SCR. (C) A wound healing assay was used to detect the effect of SLUG on JMJD2A-mediated bladder cancer cell migration ability. "P<0.05 vs. SCR. (D) Transwell invasion assay was used to detect the effect of SLUG on JMJD2A-mediated bladder cancer cell migration ability. ${ }^{*}<0.05$ vs. SCR. SCR, scramble siRNA.

with the SCR control group (Fig. 6). Consistent with the CCK- 8 assay results, in the colony formation assays, the ectopic expression of JMJD2A in T24 and 5637 cells was increased; whereas the colony formation of JMJD2A-depleted T24 and 5637 cells was decreased (Fig. 6B). These findings indicated that JMJD2A promoted the proliferation ability of bladder cancer cells.

\section{Discussion}

JMJD2A has been revealed to be aberrantly-regulated in various types of cancers, including breast $(7,8)$, bladder (9), lung $(10,11)$ and colon cancer $(12)$. In the present study, the results of RT-qPCR and western blot analyses revealed that JMJD2A was upregulated in bladder cancer (BC) tissues and cell lines, which was in contrast to a previous study (9). However, our research was consistent with a study by Kogure et al (10). The reason for the discrepancy in the results obtained may be due to the difference in the number of specimens or geographical regions of selected samples. In addition, the expression of JMJD2A was also analyzed in a bladder database on cBioPortal (http://www. cbioportal.org; data not shown), suggesting that JMJD2A is increased in bladder cancer. In order to further decipher the roles of JMJD2A in bladder cancer, CCK-8, colony formation, wound healing and Transwell invasion assays were performed, which revealed that ectopic expression of JMJD2A promoted T24 and 5637 cell proliferation, 
A

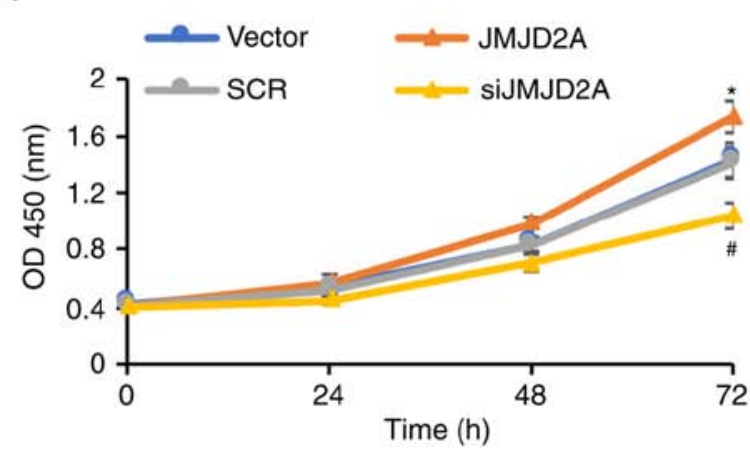

B

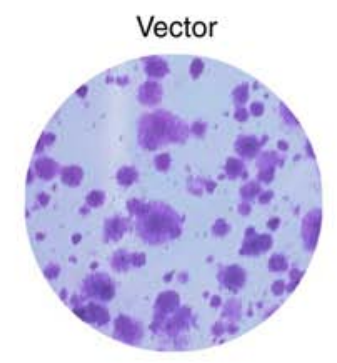

T24

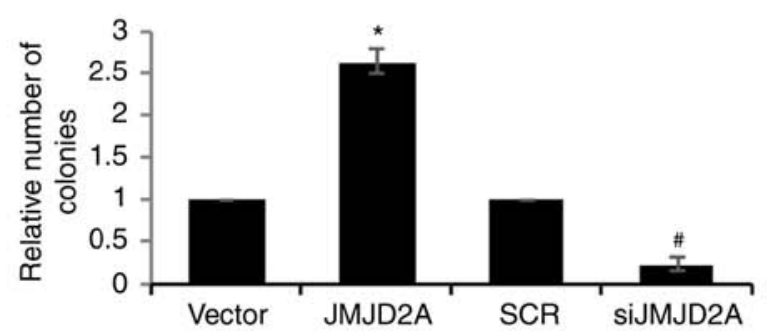

5637

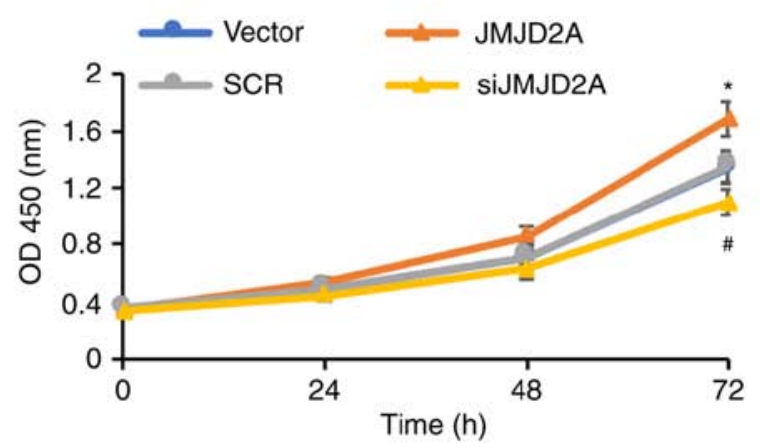

SCR
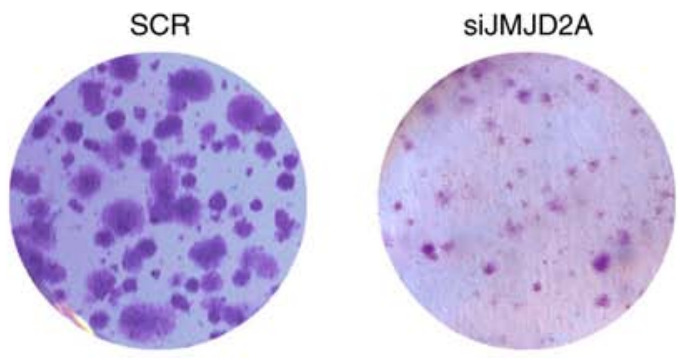

5637

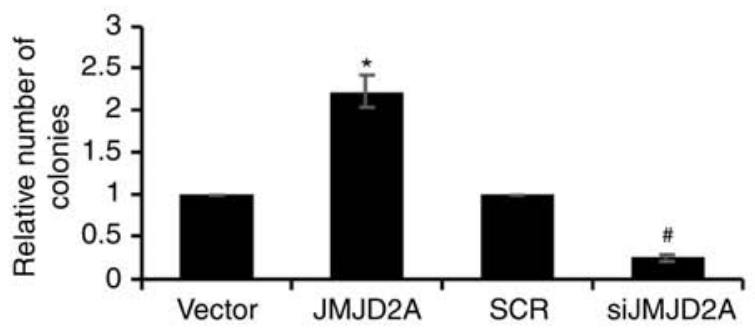

Figure 6. JMJD2A promotes bladder cancer cell proliferation. (A) JMJD2A was overexpressed or knocked down in T24 and 5637 cells, and a CCK-8 assay was performed to determine the effect of JMJD2A on cell proliferation. ${ }^{*} \mathrm{P}<0.05$ vs. vector, ${ }^{*} \mathrm{P}<0.05$ vs. SCR. (B) JMJD2A was overexpressed or knocked down in T24 and 5637 cells, and a colony formation assay was performed to determine the effect of JMJD2A on cell proliferation. The images represent the experiment in T24 cells. ${ }^{*} \mathrm{P}<0.05$ vs. vector, ${ }^{\prime} \mathrm{P}<0.05$ vs. SCR. SCR, scramble siRNA.

migration and invasion while these effects were reversed when the expression of JMJD2A was knocked down. The results demonstrated that JMJD2A could regulate bladder cancer cell functions. Moreover, Kaplan-Meier curves revealed that the patients with high expression of JMJD2A experienced poor prognosis $(\mathrm{P}<0.05)$.

One noteworthy observation was that JMJD2A promoted migration and invasion of bladder cancer cells. EMT is a well-characterized process that results in invasion and metastatic dissemination of human cancers $(21,22)$. Therefore, whether JMJD2A could modulate EMT of bladder cancer cells was further examined. Gain- and loss-of-function analysis revealed that JMJD2A overexpression decreased E-cadherin expression and increased $\mathrm{N}$-cadherin expression, while JMJD2A inhibition led to the opposite results. These data indicated that JMJD2A may modulate cell invasion by facilitating EMT in bladder cancer cells.

In the present study, our findings suggested that JMJD2A transcriptionally activated SLUG. SLUG was upregulated by JMJD2A at both the mRNA and protein level, and $S L U G$ promoter activity was significantly activated by JMJD2A, indicating a transcriptional activation of $S L U G$ by JMJD2A.
However, there are still some limitations in our study. Our research revealed that JMJD2A expression was associated with the stage of bladder cancer, and JMJD2A promoted cell proliferation, migration and invasion in bladder cancer. However, the upstream of JMJD2A in bladder cancer is still unknown. It would be interesting to decipher whether the stages of bladder cancer can also influence the expression of JMJD2A. We assume that there are some proteins which regulate JMJD2A expression in different stages of bladder cancer. In future, ATAC-seq and RNA-seq could be performed using diverse stages of bladder cancer tissue samples and potential transcription factors may be predicted. This may help us to further gain insight on bladder cancer development. Moreover, detection of the expression of JMJD2A in tissues using immunohistochemical analysis should be performed. In addition, the effect of JMJD2A in vivo should also be assessed for further investigation.

In summary, this study indicated that JMJD2A contributed to tumorigenesis in bladder cancer by regulating SLUG. Additionally, high JMJD2A expression was associated with a poor prognosis for bladder cancer patients. JMJD2A may act as an oncogene in bladder cancer and may be a potential 
therapeutic target for bladder cancer. Moreover, considering current changing clinical treatment standards, it would be of high relevance to explore the correlation between JMD2A and PD-L1 expression or other immune markers and T-cell infiltration.

\section{Acknowledgements}

Not applicable.

\section{Funding}

Not applicable.

\section{Availability of data and materials}

The datasets used during the present study are available from the corresponding author upon reasonable request.

\section{Authors' contributions}

FW, YL and GC conceived and designed the study. FW, QZ, LW, FS and BS performed the experiments. FW, QZ, FS and LW wrote the paper. FW, YL and BS reviewed and edited the manuscript. All authors read and approved the manuscript and agree to be accountable for all aspects of the research in ensuring that the accuracy or integrity of any part of the work are appropriately investigated and resolved.

\section{Ethics approval and consent to participate}

The Ethics Committee of Tengzhou Central People's Hospital (Tengzhou, China) approved the present study. All patients provided written informed consent.

\section{Patient consent for publication}

Not applicable.

\section{Competing interests}

The authors declare that they have no competing interests.

\section{References}

1. Jemal A, Bray F, Center MM, Ferlay J, Ward E and Forman D: Global cancer statistics. CA Cancer J Clin 61: 69-90, 2011.

2. Siegel RL, Miller KD and Jemal A: Cancer statistics, 2016. CA Cancer J Clin 66: 7-30, 2016.

3. Jin Y, Lu J, Wen J, Shen Y and Wen X: Regulation of growth of human bladder cancer by miR-192. Tumour Biol 36: 3791-3797, 2015.

4. Berry WL and Janknecht R: KDM4/JMJD2 histone demethylases: Epigenetic regulators in cancer cells. Cancer Res 73: 2936-2942, 2013.
5. Gray SG, Iglesias AH, Lizcano F, Villanueva R, Camelo S, Jingu H, Teh BT, Koibuchi N, Chin WW, Kokkotou E and Dangond F: Functional characterization of JMJD2A, a histone deacetylase- and retinoblastoma-binding protein. J Biol Chem 280: 28507-28518, 2005.

6. Zhang D, Yoon HG and Wong J: JMJD2A is a novel $\mathrm{N}-\mathrm{CoR}$-interacting protein and is involved in repression of the human transcription factor achaete scute-like homologue 2 (ASCL2/Hash2). Mol Cell Biol 25: 6404-6414, 2005.

7. Li BX, Zhang MC, Luo CL, Yang P, Li H, Xu HM, Xu HF, Shen YW, Xue AM and Zhao ZQ: Effects of RNA interference-mediated gene silencing of JMJD2A on human breast cancer cell line MDA-MB-231 in vitro. J Exp Clin Cancer Res 30: 90, 2011

8. Berry WL, Shin S, Lightfoot SA and Janknecht R: Oncogenic features of the JMJD2A histone demethylase in breast cancer. Int J Oncol 41: 1701-1706, 2012.

9. Kauffman EC, Robinson BD, Downes MJ, Powell LG, Lee MM, Scherr DS, Gudas LJ and Mongan NP: Role of androgen receptor and associated lysine-demethylase coregulators, LSD1 and JMJD2A, in localized and advanced human bladder cancer. Mol Carcinog 50: 931-944, 2011.

10. Kogure M, Takawa M, Cho HS, Toyokawa G, Hayashi K, Tsunoda T, Kobayashi T, Daigo Y, Sugiyama M, Atomi Y, et al: Deregulation of the histone demethylase JMJD2A is involved in human carcinogenesis through regulation of the $\mathrm{G}(1) / \mathrm{S}$ transition. Cancer Lett 336: 76-84, 2013.

11. Mallette FA and Richard S: JMJD2A promotes cellular transformation by blocking cellular senescence through transcriptional repression of the tumor suppressor CHD5. Cell Rep 2: 1233-1243, 2012.

12. Kim TD, Shin S, Berry WL, Oh S and Janknecht R: The JMJD2A demethylase regulates apoptosis and proliferation in colon cancer cells. J Cell Biochem 113: 1368-1376, 2012.

13. Bryan RT: Cell adhesion and urothelial bladder cancer: The role of cadherin switching and related phenomena. Philos Trans $\mathrm{R}$ Soc Lond B Biol Sci 370: 20140042, 2015.

14. Lamouille S, Xu J and Derynck R: Molecular mechanisms of epithelial-mesenchymal transition. Nat Rev Mol Cell Biol 15: 178-196, 2014.

15. Muramaki M, Miyake H, Terakawa T, Kumano M, Sakai I and Fujisawa M: Expression profile of E-cadherin and N-cadherin in non-muscle-invasive bladder cancer as a novel predictor of intravesical recurrence following transurethral resection. Urol Oncol 30: 161-166, 2012.

16. Han B, Cui D, Jing Y, Hong Y and Xia S: Estrogen receptor $\beta$ $(E R \beta)$ is a novel prognostic marker of recurrence survival in non-muscle-invasive bladder cancer potentially by inhibiting cadherin switch. World J Urol 32: 149-155, 2014.

17. Livak KJ and Schmittgen TD: Analysis of relative gene expression data using real-time quantitative PCR and the 2(-Delta Delta C(T)) method. Methods 25: 402-408, 2001.

18. Zhi Y, Pan J, Shen W, He P, Zheng J, Zhou X, Lu G, Chen Z and Zhou Z: Ginkgolide B inhibits human bladder cancer cell migration and invasion through microRNA-223-3p. Cell Physiol Biochem 39: 1787-1794, 2016.

19. Wan J, Zhan J, Li S, Ma J, Xu W, Liu C, Xue X, Xie Y, Fang W, Chin YE and Zhang H: PCAF-primed EZH2 acetylation regulates its stability and promotes lung adenocarcinoma progression. Nucleic Acids Res 43: 3591-3604, 2015.

20. McConkey DJ, Choi W, Marquis L, Martin F, Williams MB, Shah J, Svatek R, Das A, Adam L, Kamat A, et al: Role of epithelial-to-mesenchymal transition (EMT) in drug sensitivity and metastasis in bladder cancer. Cancer Metastasis Rev 28: 335-344, 2009.

21. Gonzalez DM and Medici D: Signaling mechanisms of the epithelial-mesenchymal transition. Sci Signal 7: re8, 2014.

22. Yun SJ and Kim WJ: Role of the epithelial-mesenchymal transition in bladder cancer: From prognosis to therapeutic target. Korean J Urol 54: 645-650, 2013. 